\title{
Red-IR stimulated luminescence in K-feldspar: Single or multiple trap origin?
}

\author{
Andersen, Martin Thalbitzer; Jain, Mayank; Tidemand-Lichtenberg, Peter
}

Published in:

Journal of Applied Physics

Link to article, DOI:

$10.1063 / 1.4745018$

Publication date:

2012

Document Version

Publisher's PDF, also known as Version of record

Link back to DTU Orbit

Citation (APA):

Andersen, M. T., Jain, M., \& Tidemand-Lichtenberg, P. (2012). Red-IR stimulated luminescence in K-feldspar: Single or multiple trap origin? Journal of Applied Physics, 112, 043507. https://doi.org/10.1063/1.4745018

\section{General rights}

Copyright and moral rights for the publications made accessible in the public portal are retained by the authors and/or other copyright owners and it is a condition of accessing publications that users recognise and abide by the legal requirements associated with these rights.

- Users may download and print one copy of any publication from the public portal for the purpose of private study or research.

- You may not further distribute the material or use it for any profit-making activity or commercial gain

- You may freely distribute the URL identifying the publication in the public portal

If you believe that this document breaches copyright please contact us providing details, and we will remove access to the work immediately and investigate your claim 


\section{AIP Appilied Physics}

\section{Red-IR stimulated luminescence in K-feldspar: Single or multiple trap origin?}

Martin Thalbitzer Andersen, Mayank Jain, and Peter Tidemand-Lichtenberg

Citation: J. Appl. Phys. 112, 043507 (2012); doi: 10.1063/1.4745018

View online: http://dx.doi.org/10.1063/1.4745018

View Table of Contents: http://jap.aip.org/resource/1/JAPIAU/v112/i4

Published by the American Institute of Physics.

\section{Related Articles}

Next generation of Ge1-ySny ( $y=0.01-0.09)$ alloys grown on Si(100) via Ge3H8 and SnD4: Reaction kinetics and tunable emission

Appl. Phys. Lett. 101, 072105 (2012)

Carrier-dopant exchange interactions in Mn-doped $\mathrm{PbS}$ colloidal quantum dots

Appl. Phys. Lett. 101, 062410 (2012)

Radiative recombination model of degenerate semiconductor and photoluminescence properties of 3C-SiC by $\mathrm{P}$ and $\mathrm{N}$ doping

J. Appl. Phys. 112, 033508 (2012)

Photoluminescence study of $\beta-\mathrm{Ga} 2 \mathrm{O} 3$ nanostructures annealed in different environments

J. Appl. Phys. 112, 034307 (2012)

Metal atom ( $\mathrm{Zn}, \mathrm{Cd}$ and $\mathrm{Mg}$ ) luminescence in solid neon

Low Temp. Phys. 38, 679 (2012)

\section{Additional information on J. Appl. Phys.}

Journal Homepage: http://jap.aip.org/

Journal Information: http://jap.aip.org/about/about_the_journal

Top downloads: http://jap.aip.org/features/most_downloaded

Information for Authors: http://jap.aip.org/authors

\section{ADVERTISEMENT}

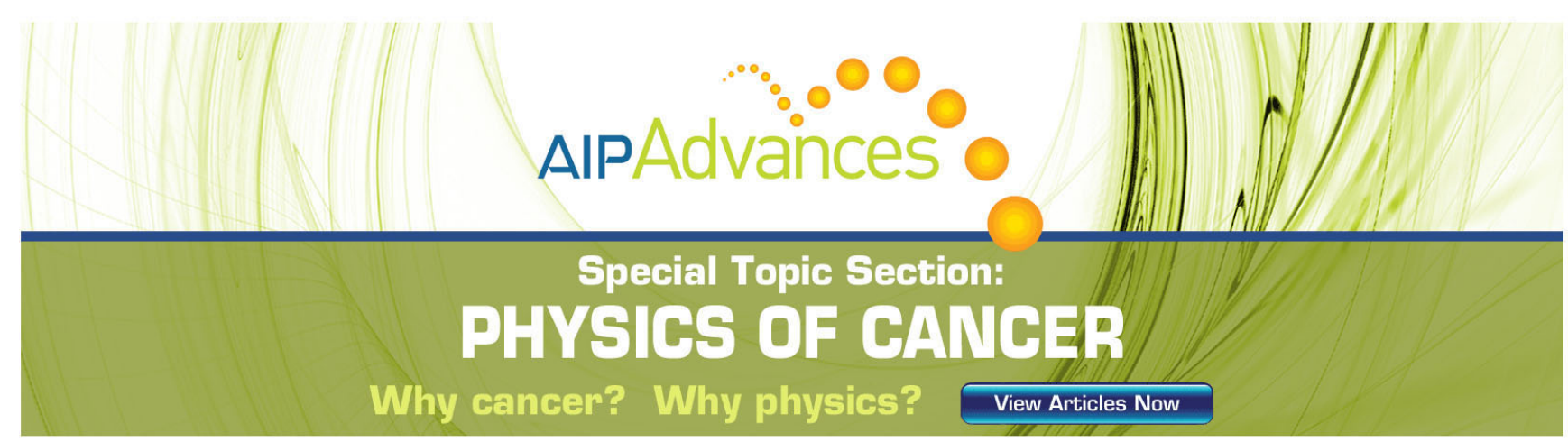




\title{
Red-IR stimulated luminescence in K-feldspar: Single or multiple trap origin?
}

\author{
Martin Thalbitzer Andersen, ${ }^{1}$ Mayank Jain, ${ }^{1, \text { a) }}$ and Peter Tidemand-Lichtenberg ${ }^{2}$ \\ ${ }^{1}$ Center for Nuclear Technologies, Technical University of Denmark, DTU Risø Campus, DK-4000 Roskilde, \\ Denmark \\ ${ }^{2}$ Department of Photonics Engineering, Technical University of Denmark, DTU Risø Campus, \\ DK-4000 Roskilde, Denmark
}

(Received 25 April 2012; accepted 11 July 2012; published online 24 August 2012)

\begin{abstract}
We investigate on the origins of the infra-red stimulated luminescence (IRSL) signals in 3 potassium feldspars based on IR-red spectroscopy $(\sim 700-1050 \mathrm{~nm})$ using a fiber-coupled tunable Ti:Sapphire laser, in combination with different thermal and optical (pre)treatments of the samples. We also measure dose-response curves with different wavelengths and at different stimulation temperatures so as to be able to distinguish between traps based on their electron trapping cross-sections. Our data suggest that the dosimetric signals, IRSL, and the post IR-IRSL in K-feldspars arise from a single electron trapping centre. (C) 2012 American Institute of Physics. [http://dx.doi.org/10.1063/1.4745018]
\end{abstract}

\section{INTRODUCTION}

Optically stimulated luminescence (OSL) and thermally stimulated luminescence (TL) signals are widely used in environmental and accident dosimetry and geochronology. OSL dating using two naturally occurring dosimeters, quartz $\left(\mathrm{SiO}_{2}\right)$, and alkali feldspars $\left(\mathrm{NaAlSi}_{3} \mathrm{O}_{8}-\mathrm{KAlSi}_{3} \mathrm{O}_{8}\right)$ has revolutionized our understanding of past climate changes, Earth surface processes, and human evolution and migration. For quartz the upper dating limit is about $150 \mathrm{ka}$, whereas feldspars have the potential to extend this limit up to about 1 Ma. ${ }^{1}$ Moreover, feldspars have the potential to extend the luminescence dating technique for in situ measurements on other planetary surfaces. ${ }^{2,3}$ Unfortunately there can be significant leakage of dosimetric information in feldspars because of rapid, time-dependent recombination from electron traps (donor states) to recombination centers (acceptor states); this process is commonly referred to as anomalous fading ${ }^{4}$ and is attributed to quantum mechanical tunneling. ${ }^{5}$ The typical fading rates in different feldspar species can vary from 0 to $20 \% /$ decade with a typical value around $5 \%,{ }^{6}$ and the presence of fading severely restricts the use of feldspars in geochronometry. Some correction methods are available, but these are based on assumptions which cannot be tested independently. ${ }^{7}$

The signal typically used in feldspar dating is called infra-red stimulated luminescence (IRSL), first reported by Hutt et al. ${ }^{8}$ who discovered a resonance peak at around $870 \mathrm{~nm}$ in the excitation spectra of feldspar. The origin of the IRSL signal has not been fully understood; some studies advocate a single-trap while others a multiple-trap origin (see a review by Duller). ${ }^{9}$ Recently a detailed model based on delocalized, semi-localized, and localized transitions has been proposed by Jain and Ankjærgaard. ${ }^{10}$ With this model, the authors have demonstrated that the key features of feldspar luminescence, both thermal and optical, can be

\footnotetext{
a) Author to whom correspondence should be addressed. Electronic mail: maja@dtu.dk.
}

explained using a single dosimetric trap when one incorporates the complex band structure ${ }^{11}$ and the spatial interaction between traps and centers in the model.

The IRSL signal has an interesting characteristic: there is an increase in the net signal intensity with an increase in the measurement temperature, and the signal can only be emptied by high temperature measurements. ${ }^{12,13}$ An important effect of this characteristic is that IRSL signal can be regenerated in consecutive measurements simply by increasing the measurement temperature. ${ }^{14-16}$ Such a regenerated signal is commonly known as post IR-IRSL (henceforth pIRIR) signal, and its measurement involves two steps, a prior IR bleach at a low temperature, e.g., $50{ }^{\circ} \mathrm{C}$ followed by IRSL measurement at some higher temperature. The pIRIR signals measured at $230^{\circ} \mathrm{C}$ or $290^{\circ} \mathrm{C}$ generally show a significantly reduced fading rate, ${ }^{1,16,17}$ and the latter has been shown to give accurate ages over the last $600 \mathrm{ka}^{1}$ It remains unresolved, however, whether the pIRIR signal originates from the same trap as the IRSL signal or from a deeper, more stable trap(s). The available data can be interpreted both in favor of multiple traps ${ }^{14,15,18}$ or a single trap. ${ }^{10,18}$

Both from the fundamental, as well as from the point of view of having a robust, reliable chronometer it is important to understand the origins of the IRSL and pIRIR signals in feldspars. It is of critical importance to know whether the IRSL signal arises from a single trap or from multiple traps. We believe that there exist conflicting views on the origin of IR stimulated signals because most interpretations are largely based on the thermal dependence the IRSL signal $^{12,14,15,18,19}$ without the corresponding spectral information. As discussed in detail by Jain and Ankjærgaard, ${ }^{10}$ thermal effects in feldspars are rather complex and therefore cannot, on their own, be used to arrive at a unique interpretation. Spectral studies can be combined with thermal and dosimetric aspects, to obtain a more robust theory on the origin of feldspar luminescence, especially with respect to single vs. multiple traps; these aspects are investigated in this contribution. 


\section{SAMPLES AND EXPERIMENTS}

Three K-feldspar samples were used: an extract from sediments (Laboratory code: $963601 \mathrm{FK}$, grain size 150-300 $\mu \mathrm{m}$ ), a museum single crystal specimen "Orthoclase 3 " and a single crystal of R28. ${ }^{11,20}$ The sample Orthoclase 3 was gently crushed into sand sized grains (grain size 90$125 \mu \mathrm{m}$ ), and three aliquots each were prepared for Orthoclase 3 and 963601 FK. Only one aliquot of R28 was prepared containing the single crystal $(\sim 1 \mathrm{~mm} \times 1 \mathrm{~mm})$.

The setup for optical stimulation consists of a continuous wave linear tunable Titanium doped Sapphire (Ti:S) laser which is pumped by a Coherent Verdi V5 laser delivering $5 \mathrm{~W}$ of $532 \mathrm{~nm}$ laser light. The Ti:S laser light is then coupled into an optical fiber (Thorlabs multimode $200 \mu \mathrm{m}$ $0.22 \mathrm{NA}$ low $\mathrm{OH}$ ) which is inserted into a custom made optical head for an automated OSL/TL dating system, Risø reader. ${ }^{21}$ This reader has an inbuilt heater plate under the sample position, and the photons emitted are counted using a photomultiplier tube. There is an built in beta $\left(\mathrm{Sr}^{90} / \mathrm{Y}^{90}\right)$ source for sample irradiation, having a dose rate of $0.1 \mathrm{~Gy} / \mathrm{s}$. The tunable laser light is used as the stimulation light source in all the measurements. In order to switch the laser light on and off a shutter was inserted in front of the fiber (Fig. 1). The laser light source was filtered using the SCHOTT RG695 (F1) and a $5 \mathrm{~mm}$ Corning 2-58 filter (F2) in order to remove the green laser light used for pumping the Ti:S laser. All luminescence emissions were detected in the blue/violet emission band using a combination of Schott BG-39 and Corning 7-59 filters (F3). The laser power was monitored with a thermal power meter (Thorlabs s302C) using a $1 \%$ beam sampler (Thorlabs BSF05-B) which was inserted right after the output from the Ti:S laser. With this setup the stimulation light (laser line width around $\sim 5 \mathrm{~nm}$ ) can be delivered with an optical power of $>300 \mathrm{~mW}$ in the wavelength range of $700 \mathrm{~nm}$ to $1000 \mathrm{~nm}$; this translates to a power density of more than $180 \mathrm{~mW} / \mathrm{cm}^{2}$ at the sample position. This optical power density is 3 to 4 orders of magnitudes larger than those used in the previous studies using a $20 \mathrm{~W}$ tungsten halogen lamp. ${ }^{11}$ We can measure OSL decay curves with a high signal-to-noise ratio, which is not possible with a lamp system.

Using this system two main types of measurements were carried out:

(1) Excitation spectra (ES): ES was studied as a function of preheat (room temperature, 150 or $250^{\circ} \mathrm{C}$ ), stimulation temperature $\left(30,230\right.$, or $290^{\circ} \mathrm{C}$ ) and prior IR wash (prior IR at $30^{\circ} \mathrm{C}$ and the post IR bleach ES measured at 230 or $290^{\circ} \mathrm{C}$ ). The two high temperatures used in our study are based on the recent work using pIRIR signals. ${ }^{1,16,17,22}$ Details of the measurement sequence is presented in Table I.

(2) Dose response curve (DRC): DRCs were measured with different IR-red stimulation wavelengths to sample different parts of the ES. DRCs were also compared at

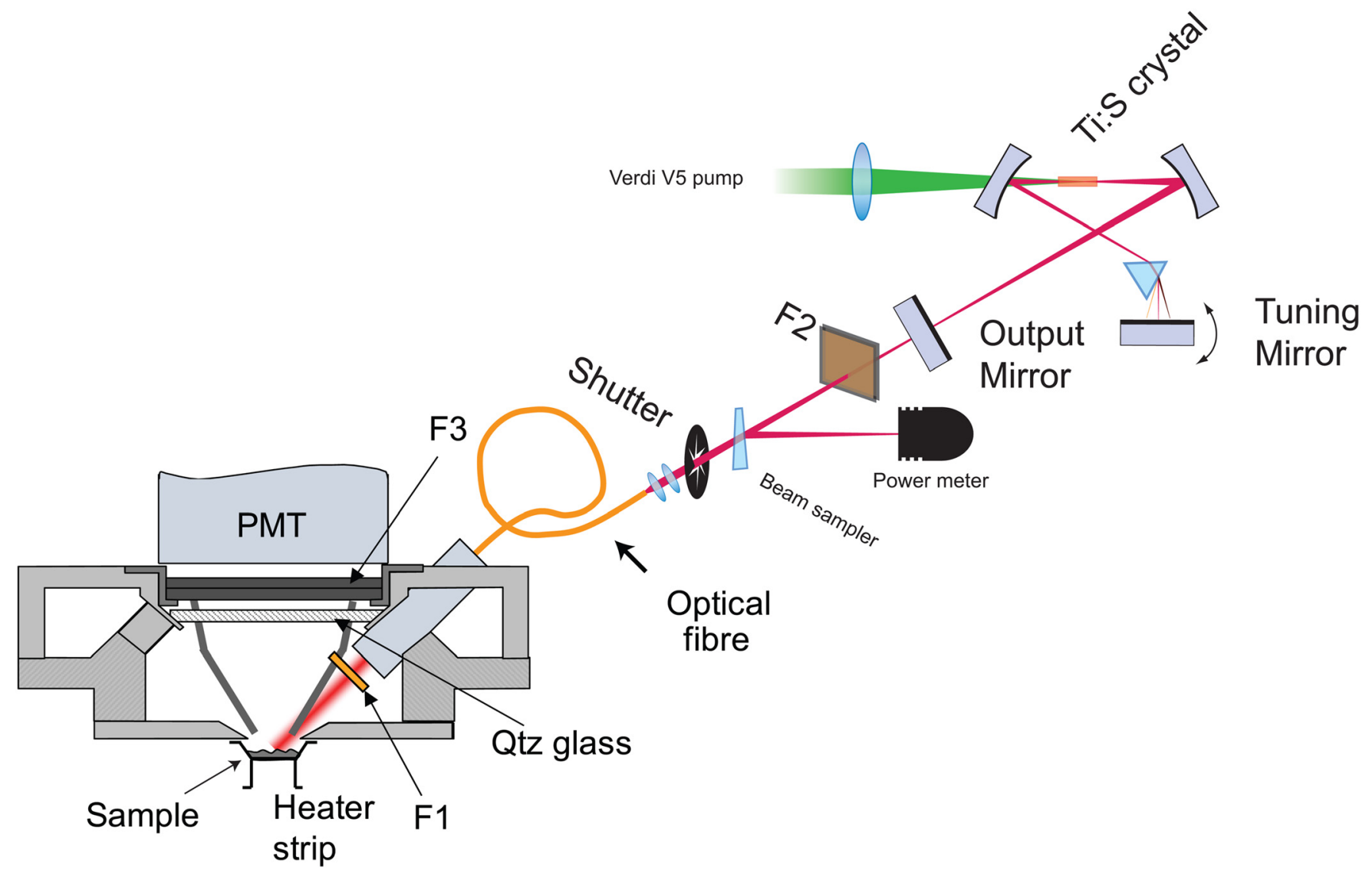

FIG. 1. Setup of the Tunable Ti doped Sapphire laser coupled to the Risø TL-OSL reader. The filters used in the setup are (F1) SCHOTT RG665, (F2) 5 mm Corning 2-58 filter, and (F3) Schott BG-39 and Corning 7-59. 
TABLE I. Sequence parameters used for measurement of ES and post IR excitation spectra (pIR-ES) for samples 963601 FK and Orthoclase 3. For sample R28 the same ES and pIR-ES protocol was used, but the dose was $720 \mathrm{~Gy}$ instead of $6 \mathrm{~Gy}$ and the heating rate for the preheat step was set to $0.2^{\circ} \mathrm{C} / \mathrm{s}$. The sequence of temperatures mentioned in the description of preheat step correspond to the same sequence as temperatures mentioned in the headings.

\begin{tabular}{|c|c|c|c|}
\hline Parameter & Preheat & $\mathrm{ES}\left(30^{\circ} \mathrm{C}, 230^{\circ} \mathrm{C}, 290^{\circ} \mathrm{C}\right)$ & pIR-ES $\left(230^{\circ} \mathrm{C}, 290^{\circ} \mathrm{C}\right)$ \\
\hline Cleanout (IRSL) & $\begin{array}{c}100 \mathrm{~s} @ 290^{\circ} \mathrm{C}(\lambda=860 \mathrm{~nm}, \\
\left.\mathrm{P}=180 \mathrm{~mW} / \mathrm{cm}^{2}\right)\end{array}$ & $\begin{array}{c}100 \mathrm{~s} @ 290^{\circ} \mathrm{C}(\lambda=860 \mathrm{~nm}, \\
\left.\mathrm{P}=180 \mathrm{~mW} / \mathrm{cm}^{2}\right)\end{array}$ & $\begin{array}{c}100 \mathrm{~s} @ 290^{\circ} \mathrm{C}(\lambda=860 \mathrm{~nm}, \\
\left.\mathrm{P}=180 \mathrm{~mW} / \mathrm{cm}^{2}\right)\end{array}$ \\
\hline$\beta$ dose & $6 \mathrm{~Gy}$ & 6Gy & $6 \mathrm{~Gy}$ \\
\hline Preheat (TL) & 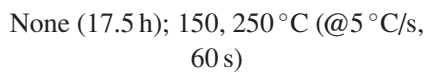 & 250,250,320C (@5C/s,60s $)$ & 250,320C (@5C/s,60s $)$ \\
\hline Bleach (IRSL) & & & $\begin{array}{c}200 \mathrm{~s} @ 30^{\circ} \mathrm{C}(\lambda=860 \mathrm{~nm} \\
\left.\mathrm{P}=180 \mathrm{~mW} / \mathrm{cm}^{2}\right)\end{array}$ \\
\hline 16xIRSL & $\begin{array}{c}24 \mathrm{~ms} @ 30^{\circ} \mathrm{C}(\lambda=1002 \mathrm{~nm}-740 \mathrm{~nm} \\
\left.\mathrm{P}=23 \mathrm{~mW} / \mathrm{cm}^{2}\right)\end{array}$ & $\begin{array}{c}24 \mathrm{~ms}(\lambda=1002 \mathrm{~nm}-740 \mathrm{~nm} \\
\left.\mathrm{P}=23 \mathrm{~mW} / \mathrm{cm}^{2}\right)\end{array}$ & $\begin{array}{c}24 \mathrm{~ms}(\lambda=1002 \mathrm{~nm}-740 \mathrm{~nm} \\
\left.\mathrm{P}=23 \mathrm{~mW} / \mathrm{cm}^{2}\right)\end{array}$ \\
\hline IRSL (Lx) & $\begin{array}{c}100 \mathrm{~s} @ 30^{\circ} \mathrm{C}(\lambda=860 \mathrm{~nm} \\
\left.\mathrm{P}=180 \mathrm{~mW} / \mathrm{cm}^{2}\right)\end{array}$ & $100 \mathrm{~s}\left(\lambda=860 \mathrm{~nm}, \mathrm{P}=180 \mathrm{~mW} / \mathrm{cm}^{2}\right)$ & $100 \mathrm{~s}\left(\lambda=860 \mathrm{~nm}, \mathrm{P}=180 \mathrm{~mW} / \mathrm{cm}^{2}\right)$ \\
\hline \multicolumn{4}{|l|}{ Depletion check } \\
\hline Cleanout (IRSL) & $\begin{array}{c}290^{\circ} \mathrm{C} 100 \mathrm{~s}(\lambda=860 \mathrm{~nm}, \\
\left.\mathrm{P}=180 \mathrm{~mW} / \mathrm{cm}^{2}\right)\end{array}$ & $\begin{array}{c}290^{\circ} \mathrm{C} 100 \mathrm{~s}(\lambda=860 \mathrm{~nm}, \\
\left.\mathrm{P}=180 \mathrm{~mW} / \mathrm{cm}^{2}\right)\end{array}$ & $\begin{array}{l}290^{\circ} \mathrm{C} 100 \mathrm{~s}(\lambda=860 \mathrm{~nm} \\
\left.\mathrm{P}=180 \mathrm{~mW} / \mathrm{cm}^{2}\right)\end{array}$ \\
\hline$\beta$ dose & 6 Gy & $6 \mathrm{~Gy}$ & $6 \mathrm{~Gy}$ \\
\hline Preheat (TL) & $\begin{array}{c}\mathrm{TL} \text { none, } 150^{\circ} \mathrm{C}, 250^{\circ} \mathrm{C}\left(@ 5^{\circ} \mathrm{C} / \mathrm{s} \text {, }\right. \\
60 \mathrm{~s})\end{array}$ & $250,250,320^{\circ} \mathrm{C}\left(@ 5^{\circ} \mathrm{C} / \mathrm{s}, 60 \mathrm{~s}\right)$ & $250^{\circ} \mathrm{C}, 320^{\circ} \mathrm{C}\left(@ 5^{\circ} \mathrm{C} / \mathrm{s}, 60 \mathrm{~s}\right)$ \\
\hline IRSL (Tx) & $\begin{array}{c}100 \mathrm{~s} @ 30^{\circ} \mathrm{C}(\lambda=860 \mathrm{~nm} \\
\left.\mathrm{P}=180 \mathrm{~mW} / \mathrm{cm}^{2}\right)\end{array}$ & $100 \mathrm{~s}\left(\lambda=860 \mathrm{~nm}, \mathrm{P}=180 \mathrm{~mW} / \mathrm{cm}^{2}\right)$ & $100 \mathrm{~s}\left(\lambda=860 \mathrm{~nm}, \mathrm{P}=180 \mathrm{~mW} / \mathrm{cm}^{2}\right)$ \\
\hline
\end{tabular}

different temperatures using IR and pIRIR signals using $860 \mathrm{~nm}$ as stimulation wavelength. Experimental details are given in Tables II and III.

(3) Correlation between decay constant and initial intensity: OSL decay curves were measured using different wavelengths $(740-1000 \mathrm{~nm})$ after the same dose and preheat. The OSL decay constant and initial intensity were then estimated by exponential fitting of the data for the first $5 \mathrm{~s}$.

For ES measurements an optical power of around 30-50 $\mathrm{mW}$ was used for each excitation wavelength using an exposure time of $\sim 24 \mathrm{~ms}$; this translates to a power density of $20-30 \mathrm{~mW} / \mathrm{cm}^{2}$ at the sample position Thus the optical energy per measurement point was around 0.4$0.6 \mathrm{~mJ}$, and each measurement sequence contained 16 exposures to uniformly cover the wavelength range (740$1000 \mathrm{~nm}$ ) with a resolution of $\sim 10 \mathrm{~nm}$ around the peak and $\sim 20 \mathrm{~nm}$ for the sidebands. This approach ensured that the entire scanning range was covered with a reasonable resolution and a good signal-to-noise ratio (SNR), while restricting the overall OSL signal depletion to less than $10 \%$. The net optical depletion was routinely checked

TABLE II. Parameters used for measuring the wavelength dependence of dose response curves for samples $963601 \mathrm{FK}$ and R28.

\begin{tabular}{lc}
\hline \hline SAR cycle & Steps \\
\hline$\beta$ dose & $0-2000 \mathrm{~Gy}$ \\
Preheat (TL) & $250{ }^{\circ} \mathrm{C} @\left(2{ }^{\circ} \mathrm{C} / \mathrm{s}, 60 \mathrm{~s}\right)$ \\
IRSL (Lx) & $250 \mathrm{~s} @ 30^{\circ} \mathrm{C}$ \\
Cleanout & $250 \mathrm{~s} @ 225^{\circ} \mathrm{C}$ \\
$\beta$ dose & $100 \mathrm{~Gy}$ \\
Preheat (TL) & $250{ }^{\circ} \mathrm{C}, @\left(2{ }^{\circ} \mathrm{C} / \mathrm{s}, 60 \mathrm{~s}\right)$ \\
IRSL (Tx) & $250 \mathrm{~s} @ 30^{\circ} \mathrm{C}$ \\
Cleanout OSL & $100 \mathrm{~s} @ 290^{\circ} \mathrm{C}$ \\
\hline
\end{tabular}

using a long IRSL measurement at the end of each ES sequence.

For DRC measurements optical stimulation was carried out until the signal reached an almost flat background. The time required to achieve this varied from wavelength to wavelength; with the available power $200 \mathrm{~s}$ of stimulation was found to be sufficient for all the wavelengths.

\section{RESULTS AND DISCUSSION}

\section{A. Excitation spectra measurements}

Temperature dependent excitation spectra (ES) for feldspar samples have previously been studied by Poolton et al. ${ }^{11}$ at temperatures $10-300 \mathrm{~K}$ and by Baril and Huntley ${ }^{23}$ at temperatures 290-490 K. Baril and Huntley concluded that there was evidence for at least two peaks at $\sim 1.45 \mathrm{eV}$ (dominant peak) and $\sim 1.57 \mathrm{eV}$ in most of their samples, although, for some samples it was found necessary to include a minor peak at $\sim 1.3 \mathrm{eV}$ to obtain the best fit using a sum of Voigt, Gaussian, and exponential functions. Poolton et al. fitted their ES to peaks at $1.46 \mathrm{eV}$ (main peak), $1.56 \mathrm{eV}$ and $1.24 \mathrm{eV}$ using multiple Gaussians. There seems to be a good agreement between these values and those of Baril and Huntley; one can thus safely assume that these IR resonance peaks at $\sim 1.46$ and $\sim 1.56 \mathrm{eV}$ are characteristic of feldspar samples. However, these data are not conclusive of whether there is a single trap or multiple traps that give rise to the excitation spectra. Baril and Huntley also examined the correlation between shape and intensity of the OSL signals obtained at different wavelengths. Their data suggests a single trap at the depth of $>2.5 \mathrm{eV}$ below the conduction band, having 2 or 3 excited states; however, this analysis on its own has inherent assumptions (discussed later) and, therefore, additional data is necessary to support or falsify the interpretation. Sanderson ${ }^{24}$ argued that a distribution of traps 
TABLE III. Parameter used measuring the temperature dependence for dose response curves. The sequence of temperatures mentioned in the description of preheat and cleanout steps correspond to the same sequence as temperatures mentioned in the headings.

\begin{tabular}{lcr}
\hline \hline SAR cycle & $\mathrm{IR}\left(50^{\circ} \mathrm{C}, 230^{\circ} \mathrm{C}, 290^{\circ} \mathrm{C}\right)$ & $\mathrm{pIRIR}\left(230^{\circ} \mathrm{C}, 290^{\circ} \mathrm{C}\right)$ \\
\hline$\beta$ dose & $0-2000 \mathrm{~Gy}$ & $0-2000 \mathrm{~Gy}$ \\
Preheat (TL) & $250,250,320^{\circ} \mathrm{C} @\left(5^{\circ} \mathrm{C} / \mathrm{s}, 60 \mathrm{~s}\right)$ & $250,320^{\circ} \mathrm{C} @\left(5^{\circ} \mathrm{C} / \mathrm{s}, 60 \mathrm{~s}\right)$ \\
IRSL $(\mathrm{Lx})$ & $200 \mathrm{~s} @ 30^{\circ} \mathrm{C}, 230^{\circ} \mathrm{C}, 290^{\circ} \mathrm{C}$ & $200 \mathrm{~s} @ 30^{\circ} \mathrm{C}$ \\
pIRIR & $200 \mathrm{~s} @ 230^{\circ} \mathrm{C}$, none, none & $200 \mathrm{~s} @ 230^{\circ} \mathrm{C}, 290^{\circ} \mathrm{C}$ \\
Cleanout & $100 \mathrm{~Gy}$ & $100 \mathrm{~Gy}$ \\
$\beta$ dose & $250,250,320^{\circ} \mathrm{C} @\left(5^{\circ} \mathrm{C} / \mathrm{s}, 60 \mathrm{~s}\right)$ & $250^{\circ} \mathrm{C}, 320^{\circ} \mathrm{C} @\left(5^{\circ} \mathrm{C} / \mathrm{s}, 60 \mathrm{~s}\right)$ \\
Preheat (TL) & $200 \mathrm{~s} @ 30,230,290^{\circ} \mathrm{C}$ & $200 \mathrm{~s} @ 30^{\circ} \mathrm{C}$ \\
IRSL & $100 \mathrm{~s} @ 290^{\circ} \mathrm{C}, 290^{\circ} \mathrm{C}$, none & $200 \mathrm{~s} @ 230^{\circ} \mathrm{C}, 290^{\circ} \mathrm{C}$ \\
pIRIR (Tx) & & $100 \mathrm{~s} @ 290^{\circ} \mathrm{C}, \mathrm{none}$ \\
Cleanout OSL &
\end{tabular}

at both shallow and deeper levels contribute to the luminescence signal; this argument is supported by (a) the shape of IRSL pulse anneal curves where a continuous depletion in the IRSL signal intensity with preheat is observed even around room temperature, and (b) there is a reduction in both the low temperature and high temperature TL peaks after an IR shine on the sample. ${ }^{9,19}$ The straight forward interpretation of these data is that there are multiple electron traps participating in the IRSL production. A single trap model can also explain these results if the decrease in luminescence signal is because of the depletion in the proximal hole population. ${ }^{10}$ The existing data cannot resolve the issue. Similarly, there is also a considerable debate on the origin of pIRIR signal; it has not yet been established experimentally whether the pIRIR signal arises from the same trap(s) as the IRSL signal or from a different (set of) trap(s).

Excitation spectroscopy is a powerful tool that can potentially resolve these debates. We present in the following sections ES as a function of preheat and stimulation temperature, and a comparative analysis of ES with or without a prior IR bleach to gain better insight into the origins of IRSL and pIRIR signals.

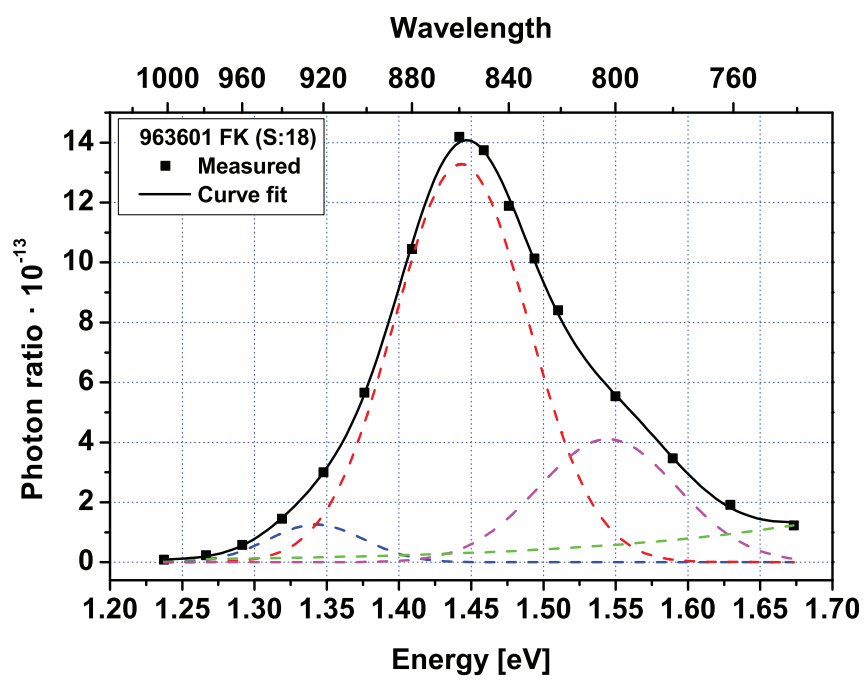

FIG. 2. Curve fitting of the excitation spectra of sample 963601 FK (aliquot 18) using multiple Gaussians peaks and a rising exponential (dashed curves). Photon ratio is estimated by dividing the total measured luminescence counts by the total number of stimulating photons at each wavelength. The positions of the fitted Gaussian peaks are at 1.34, 1.44, and $1.54 \mathrm{eV}$.
In order to do these comparisons we first need to characterize the excitation spectra of our sample. We measured ES for all our samples after a dose of $6 \mathrm{~Gy}$, except for R28 which received $720 \mathrm{~Gy}$, followed by a preheat of $250{ }^{\circ} \mathrm{C}$ and observed very similar, asymmetric spectra as have been reported in literature (e.g., Baril and Huntley, 2002). The spectral analysis of sample 963601 FK is shown in Fig. 2; this is best described by a rising continuum exponential and a sum of three Gaussian peaks. The dominant peak is centered at $1.44 \mathrm{eV}$, while there are two smaller peaks at 1.34 and $1.54 \mathrm{eV}$. The results for the other samples are similar and summarized in Table IV. These values are consistent with those reported in literature. We also attempted other combinations at different energies and using Voigt function instead of Gaussian. Our attempts suggested that at least three peaks are required to explain the data but the exact combination of peak energies and amplitudes is not unique at least in terms of fitting analysis. Because of this limitation in fitting we chose to interpret our data without depending on spectral analysis, purely analyzing our data in terms of peak shift or thermal broadening of the spectral data.

\section{Preheat dependence of ES}

If the IR-red ES arises from traps at different depths then a shape change should be observed with prior heating (preheat) because of a preferential depletion in the electrons trapped at shallower depths.

Samples were irradiated with $6 \mathrm{~Gy}$ of beta dose and the preheat dependence was examined for preheats at room temperature $\left(\sim 25^{\circ} \mathrm{C}\right), 150$ or $250{ }^{\circ} \mathrm{C}$. In the room temperature case, the sample was stored for $17.5 \mathrm{~h}$ before measurements were carried out in order to avoid any prompt luminescence decay. In the other two cases the sample was kept at either at 150 or $250{ }^{\circ} \mathrm{C}$ for $60 \mathrm{~s}$ before the ES measurements. The measurement sequence can be found in Table I.

The results for 963601 FK are shown in Fig. 3. Interestingly the peak intensity reduces with preheat (Fig. 3(a)), but peak shape and peak position are invariant (Fig. 3(b)). A similar trend is observed in the sample Orthoclase 3 (Figs. 3(c) and 3(d)).

If the IR-red ES arises from individual resonances of a continuum of traps with different activation energies as proposed by Sanderson, it will be reasonable to expect that the 
TABLE IV. Gaussian fitting parameter for excitation spectra performed at $300 \mathrm{~K}$ for each aliquot.

\begin{tabular}{|c|c|c|c|c|c|c|}
\hline \multirow[b]{2}{*}{ Sample } & \multicolumn{2}{|c|}{ Peak 1} & \multicolumn{2}{|c|}{ Peak 2} & \multicolumn{2}{|c|}{ Peak 3} \\
\hline & Energy[eV] & Area[\%] & Energy[eV] & Area[\%] & Energy $[\mathrm{eV}]$ & Area[\%] \\
\hline Orthoclase 3 (2) & $1.37 \pm 0.28$ & 11.0 & $1.44 \pm 0.02$ & 60.6 & $1.54 \pm 0.05$ & 28.4 \\
\hline Orthoclase 3 (4) & $1.41 \pm 0.20$ & 29.4 & $1.44 \pm 0.004$ & 39.6 & $1.54 \pm 0.02$ & 31.0 \\
\hline Orthoclase 3 (6) & $1.36 \pm 0.09$ & 10.0 & $1.45 \pm 0.01$ & 66.4 & $1.55 \pm 0.05$ & 23.6 \\
\hline 963601 FK (14) & $1.34 \pm 0.02$ & 5.0 & $1.44 \pm 0.005$ & 74.4 & $1.55 \pm 0.03$ & 20.6 \\
\hline 963601 FK (16) & $1.37 \pm 0.06$ & 10.6 & $1.44 \pm 0.007$ & 61.5 & $1.54 \pm 0.02$ & 27.9 \\
\hline 963601 FK (18) & $1.34 \pm 0.02$ & 4.9 & $1.44 \pm 0.004$ & 72.0 & $1.54 \pm 0.02$ & 23.1 \\
\hline R28 & $1.37 \pm 0.004$ & 5.3 & $1.47 \pm 0.003$ & 88.1 & $1.61 \pm 0.003$ & 6.6 \\
\hline
\end{tabular}

ES will change shape as a function of preheat since shallow traps with low activation energies would be affected to a relatively greater extent by preheat than traps with higher activation energies. This effect is expected to give rise to an increasingly steep slope in the low energy part of the ES, and a shift in the low energy cut-off range. These effects are, however, not observed in our data. The ES shape is invariant with preheat falsifying the hypothesis that shallow traps play any role in the origin of the IRSL signal.
Our results suggest that the source of IRSL is either a single deep trap or several deep traps whose relative population is not changed by our maximum preheat of $250{ }^{\circ} \mathrm{C}$ for $60 \mathrm{~s}$. Later, we present the results for ES measured after a preheat at $290^{\circ} \mathrm{C}$ and $320^{\circ} \mathrm{C}$ (Fig. 4); again, the shape of these data is similar to the data obtained with lower preheats despite a significant reduction in the peak intensity, suggesting that the relevant trap responsible for the IRSL signal is not eroded by $320^{\circ} \mathrm{C}$ preheat. The invariance of shape even
Wavelength [nm]
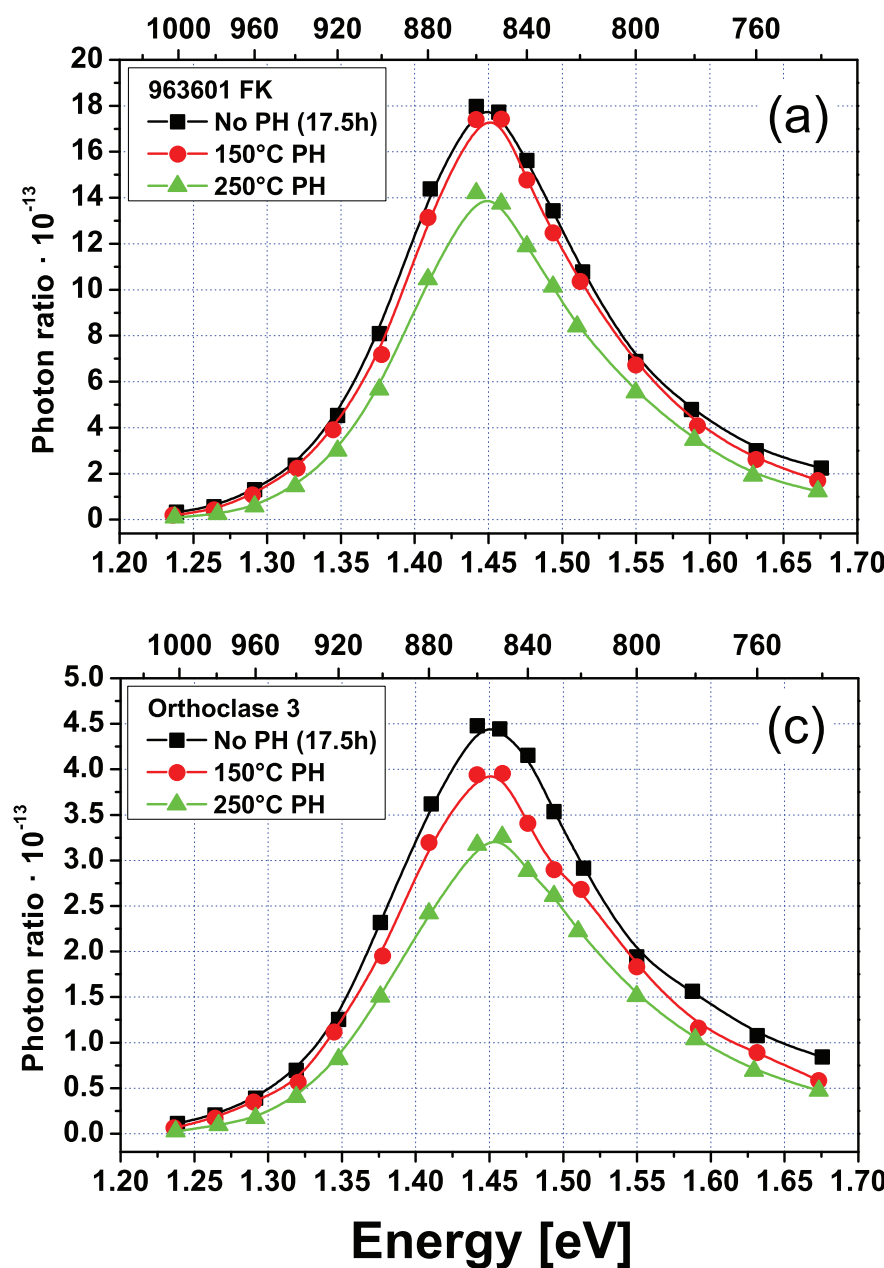

Wavelength [nm]
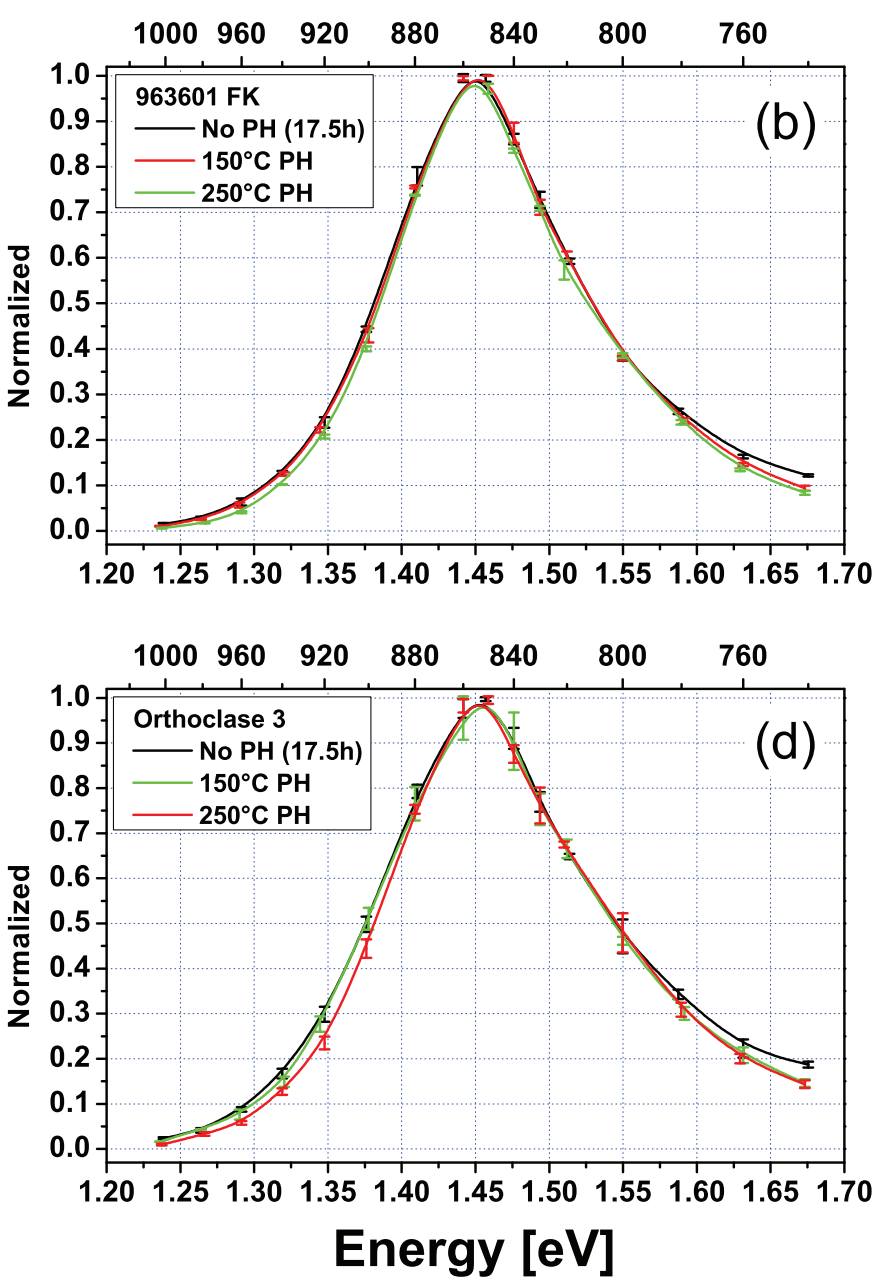

FIG. 3. The influence of preheat on the excitation spectra of sample 963601 FK: (a) Photon ratio is estimated by dividing the total luminescence counts by the total number of stimulating photons, (b) normalized counts estimated by dividing the photon ratio at different wavelengths by the peak value. The influence of preheat on Orthoclase 3: (c) photon ratio, (d) normalized counts. 

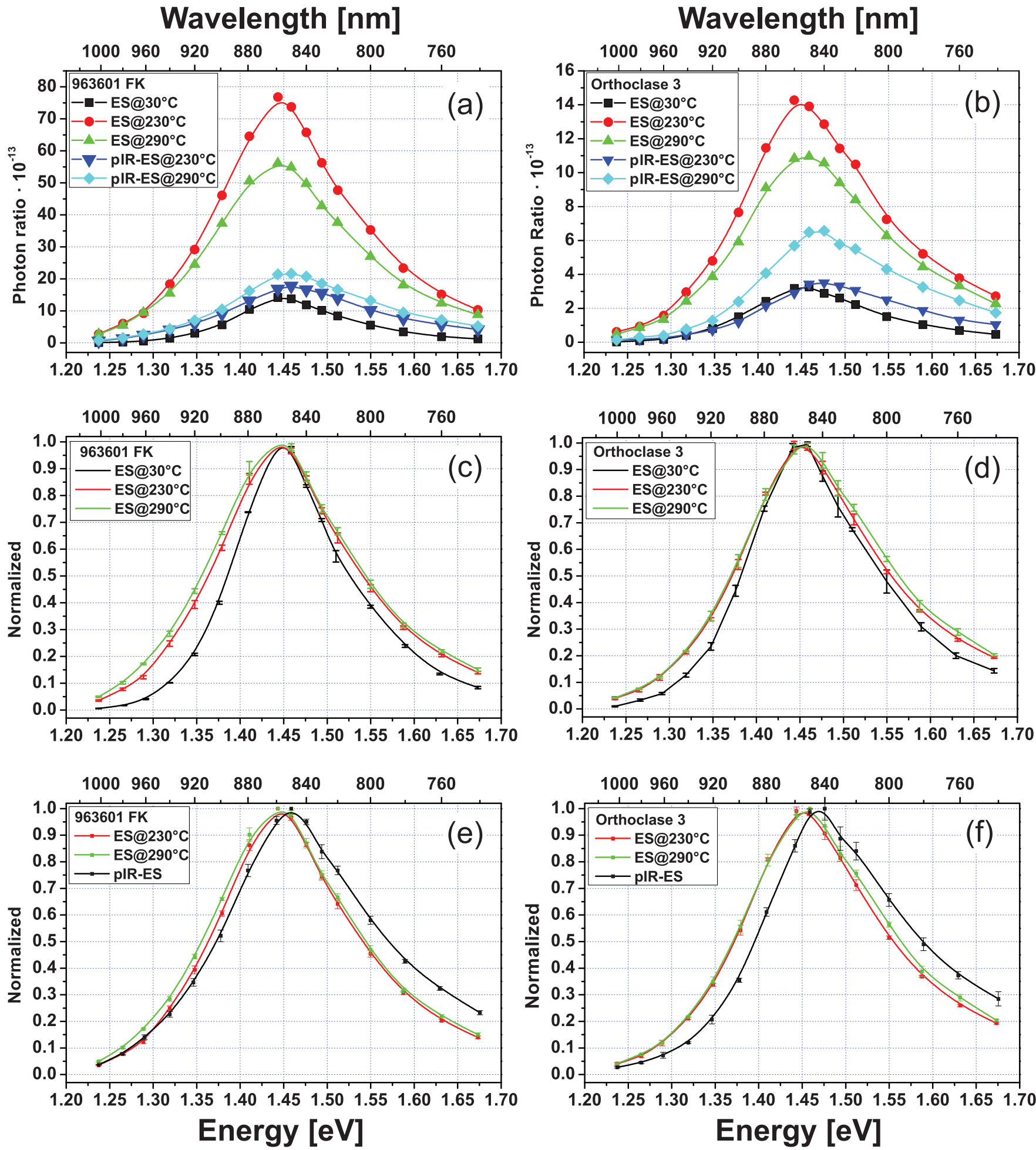

FIG. 4. Excitation spectra measured without a prior IR bleach (ES 30, 230 and $290^{\circ} \mathrm{C}$ ) or with a prior IR bleach (pIR-ES 230 and $290^{\circ} \mathrm{C}$ ) in samples $963601 \mathrm{FK}$ (left column) and Orthoclase 3 (right column). The photon ratio is estimated by dividing the total measured luminescence counts by the total number of stimulating photons. The photon ratio is plotted in (a) and (b), while normalized counts (estimated by dividing the photon ratio at different wavelengths by the peak value) are plotted in (c) and (d). Combined averaged pIR-ES $\left(230\right.$ and $\left.290^{\circ} \mathrm{C}\right)$ together with the corresponding high temperature ES are plotted in (e) and (f).

for higher preheat temperatures likely suggests that only one trap is participating in the IRSL process. These results support the interpretation of Murray et al. ${ }^{19}$ that the source of the IRSL in K-feldspars is a common trap that gives rise to the TL peak situated at about $400{ }^{\circ} \mathrm{C}$ and are consistent with the feldspar model presented by Jain and Ankjærgaard. ${ }^{10}$
If all the ES arise from a single deep trap then one can question why there is a decrease in the peak intensity with preheat. The likely explanation for this effect is a reduction in the population of the potential recombination sites during preheat because of recombination of electrons from, for example, (a) shallow, non IR sensitive states and/or (b) IR 
resonant states that have a nearby recombination centre. Our data thus provides a direct experimental evidence for the deep trap origin of IRSL as argued by Murray et al. ${ }^{19}$ These data however do not completely establish whether IRSL originates from a single or multiple deep traps. This issue is investigated in the following sections.

\section{Stimulation temperature dependence of ES}

ES of $963601 \mathrm{FK}$ and Orthoclase 3 were measured at stimulation temperatures 30,230 and $290^{\circ} \mathrm{C}$ after irradiation and preheat. The samples $963601 \mathrm{FK}$ and Orthoclase 3 both received a dose of $6 \mathrm{~Gy}$ followed by a preheat of $250^{\circ} \mathrm{C}$ for $60 \mathrm{~s}$ prior to $\mathrm{ES}$ measurements at $30^{\circ} \mathrm{C}$ or $230^{\circ} \mathrm{C}$, or a preheat of $320^{\circ} \mathrm{C}$ for $60 \mathrm{~s}$ prior to ES measurement at $290^{\circ} \mathrm{C}$. The measurement protocol is summarized in Table $\mathrm{I}$.

The data from one aliquot of $963601 \mathrm{FK}$ and one of Orthoclase 3 are presented in Figs. 4(a) and 4(b). A general trend is observed that an increase in stimulation temperature increases the intensity at all wavelengths; the peak intensity at $290^{\circ} \mathrm{C}$ is actually slightly lower than that at $230^{\circ} \mathrm{C}$, but this is due to the difference in the preheat temperatures for the two signals. The normalized (by peak intensity) averaged ES spectra for the three aliquots each of the samples 963601 FK and Orthoclase 3 are presented in Figs. 4(c) and 4(d). The increase in intensity as function of temperature occurs evenly across the resonance such that the peak position is invariant, but there is a slight broadening of the spectral width. Baril and Huntley ${ }^{23}$ have also examined temperature dependence of the ES from about $140-220^{\circ} \mathrm{C}$. It is difficult to assess peak change shapes from their data as they are not normalized, but based on fitting of the ES they concluded that peak broadening for the main resonance at $1.44 \mathrm{eV}$ are rather small (10-20 meV).

At least to the first order, these data point towards a single trap origin of ES since if there were several deep traps giving rise to the spectra, then it is expected that the temperature dependence of the individual traps will be slightly different because of the different activation ener- gies associated with the different trap depths; such an effect is expected to cause a significant change in the ES peak shape and/or position. Based on our data we can therefore conclude that the majority of the signal arises from a single trap. For a minor proportion of the signal, one could alternatively argue that the slight peak broadening is not just simple thermal broadening, but it could arise from a slight relative increase in the amplitudes of the side peaks, thus opening up the question of multiple trap origin for these side peaks. This question is further resolved in the following sections.

In either case it is interesting that there is a significant increase in the signal intensity of the main peak with temperature without a shift in the peak position with temperature. This data support that an increase in the total IRSL $(\sim 860 \mathrm{~nm})$ is due to an increase in the recombination probability because of greater mobility at higher temperatures and not due to presence of a deeper trap that becomes more active. ${ }^{10}$ The latter case will have caused a peak shift.

\section{Post IR excitation spectra}

The measurement sequence is the same as that for temperature dependent ES, however a IR $(860 \mathrm{~nm})$ bleach for $200 \mathrm{~s}$ at $30^{\circ} \mathrm{C}$ is inserted before the ES measurement at $230^{\circ} \mathrm{C}$ or $290^{\circ} \mathrm{C}$ (Table I) The pIR-ES are shown in Figs. 4(a) and 4(b) for both 963601 FK and Orthoclase 3.

The pIR-ES have a considerably reduced intensity than the corresponding ES measured without any prior IR at the same temperatures. After normalization for peak intensity the pIR-ES for both the $230^{\circ} \mathrm{C}$ and $290^{\circ} \mathrm{C}$ were found to be indistinguishable from each other. Therefore for presentation clarity the average of the two results is presented as a single pIR-ES in Figs. 4(e) and 4(f) together with the corresponding high temperature ES. These data suggest that the peak of pIR-ES are blue shifted in comparison to the high temperature ES by $\sim 10 \mathrm{meV}$ for $963601 \mathrm{FK}$ and by $\sim 15-20 \mathrm{meV}$ for Orthoclase 3 .

The same comparison was also done using the R28 Kfeldspar sample. The data are shown in Fig. 5. Although the
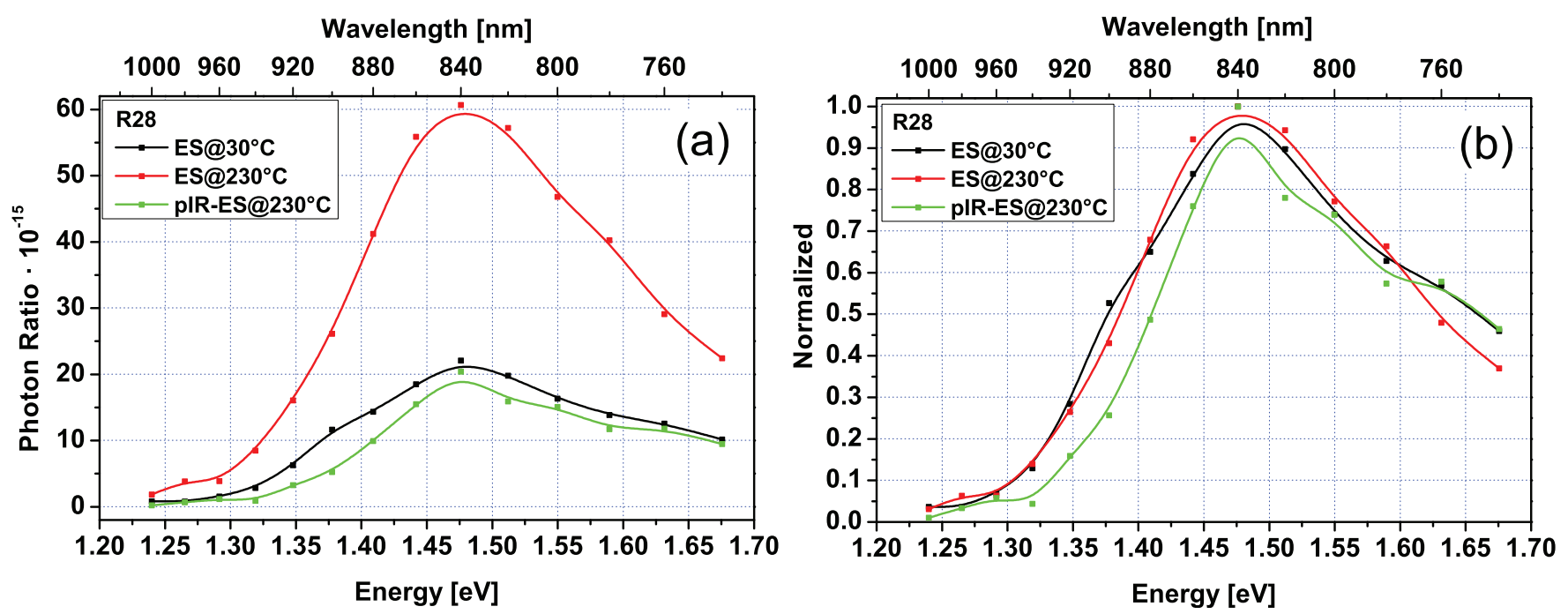

FIG. 5. (a) Excitation spectra and post IR excitation spectra of R28 K-feldspar sample. (b) Same data as (a) normalized to the peak intensity. 

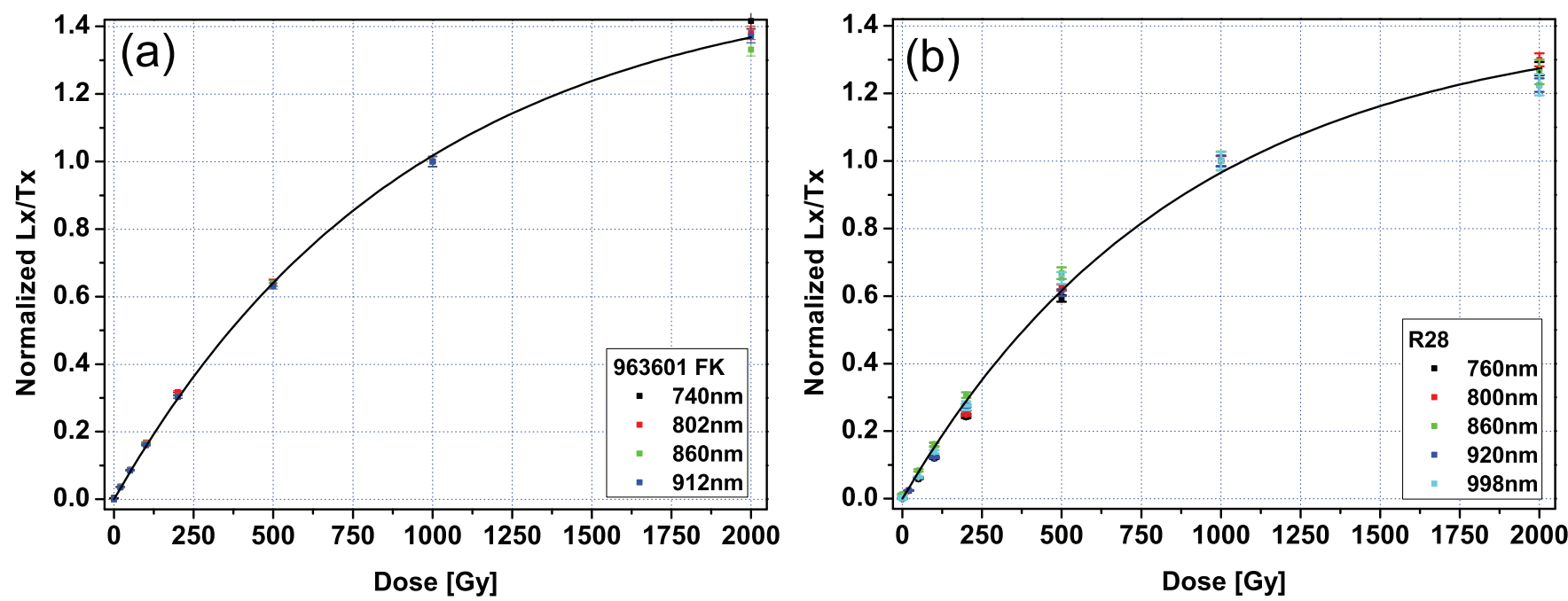

FIG. 6. (a) Dose response curves for sample $963601 \mathrm{FK}$ at different wavelengths covering $740-912 \mathrm{~nm}$ normalized to Lx/Tx signal at $1000 \mathrm{~Gy}$ for each wavelength. (b) Dose response curves for sample R28 at different wavelengths covering 760-998 nm normalized to the Lx/Tx signal at $1000 \mathrm{~Gy}$ for each wavelength.

data is noisier than the other two samples because of low OSL intensity from this sample, it appears that blue shift is not present in this sample. Moreover, the ES peak seems to occur at a slightly higher energy $1.47 \mathrm{eV}(845 \mathrm{~nm})$ compared to $1.45 \mathrm{eV}$ $(855 \mathrm{~nm})$ for the $963601 \mathrm{FK}$ and Orthoclase 3 samples.

The most interesting aspect of these data is that the pIRES of the samples are very similar to the ES measured at higher temperatures discussed in the previous section. Moreover, we do not see any gradual shift in the peak energy for example between 30,230 , and $290^{\circ} \mathrm{C}$ ES data or between 230 and $290^{\circ} \mathrm{C}$ pIR-ES data. There only exists a discrete shift between ES and pIR-ES. Furthermore pIR-ES measurements show that the ES measured at high temperatures consists largely of a signal that can be measured/emptied at $30{ }^{\circ} \mathrm{C}\left(\sim 60 \%-80 \%\right.$ for the $230{ }^{\circ} \mathrm{C}$ measurement and $\sim 40 \%-$ $60 \%$ for $290^{\circ} \mathrm{C}$ ), but also a signal that is not emptied by the low temperature IR bleach. There can be two interpretations of these data:

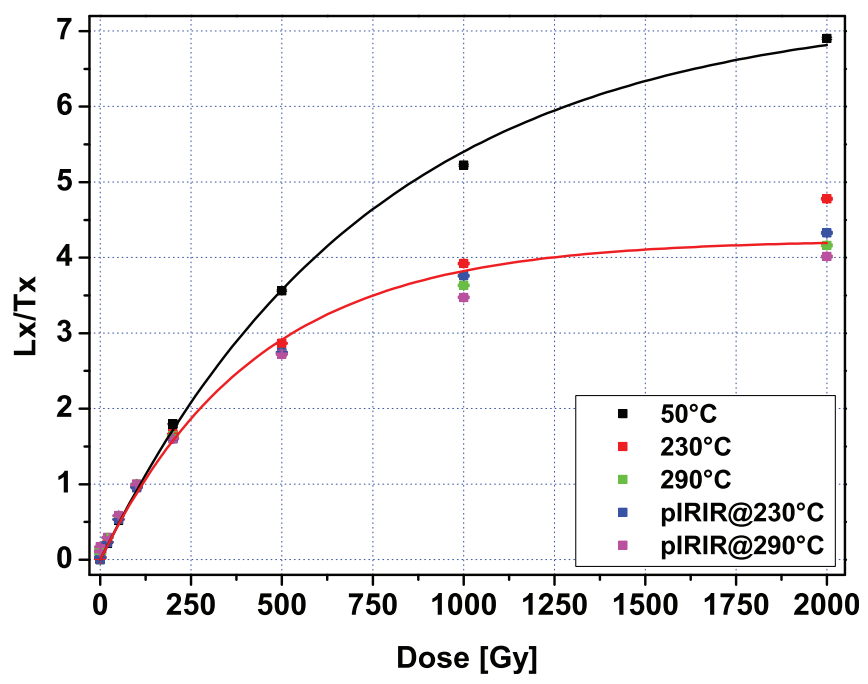

FIG. 7. IR post IR IRSL dose response curves measured using $860 \mathrm{~nm}$ at different temperatures in sample 963601 FK. All high temperature measurement.
(1) Multiple traps: there are at least two different traps that give rise to the IR and pIRIR signals. The energy barrier (example ground state to excited state, or between excited state to the band tail states) for luminescent recombination differs by about $10-20 \mathrm{meV}$ in these traps. Existence of two sets of traps has been suggested previously by other workers. ${ }^{12,14,15,25}$ However, this hypothesis does not explain why the peak does not shift gradually for different temperatures in the high temperature ES data. There should have been a detectable shift, since the pIR-ES signal comprises a significant proportion of the ES signal. Moreover, this hypothesis does not explain why there is no peak shift in R28 and why there is a large difference in the thermal stabilities observed in the pulse anneal curves for the two signals which requires 100 's of $\mathrm{meV}$ for explanation rather than a 10's of meV observed in the spectral data. ${ }^{14,15,18}$ Based on these considerations we rule out the possibility of multiple trap origin.

(2) Single trap: The data can be explainable by a single dosimetric trap. Here the low temperature stimulation empties all or most of the trapped electrons that have a nearby recombination centre. As the close by recombination centers are used it is necessary for the evicted electron to occupy a higher energy state in the band tail states to achieve the mobility required for recombination with a distant hole. This additional activation energy required for recombination causes the blue shift in the pIR-ES. Based on time-resolved OSL estimation of the activation energy for such recombination was found to be of the order of $10-20 \mathrm{meV}$ corresponding to phonon vibrational modes of $5-6 \mathrm{meV},{ }^{10}$ these estimates correspond closely to the blue shift observed here in the spectral data. Based on this reasoning the absence of peak shift in R28 could in principle be explained by a hypothetical larger density of holes; thus, the phonon assistance is not necessary for recombination. 

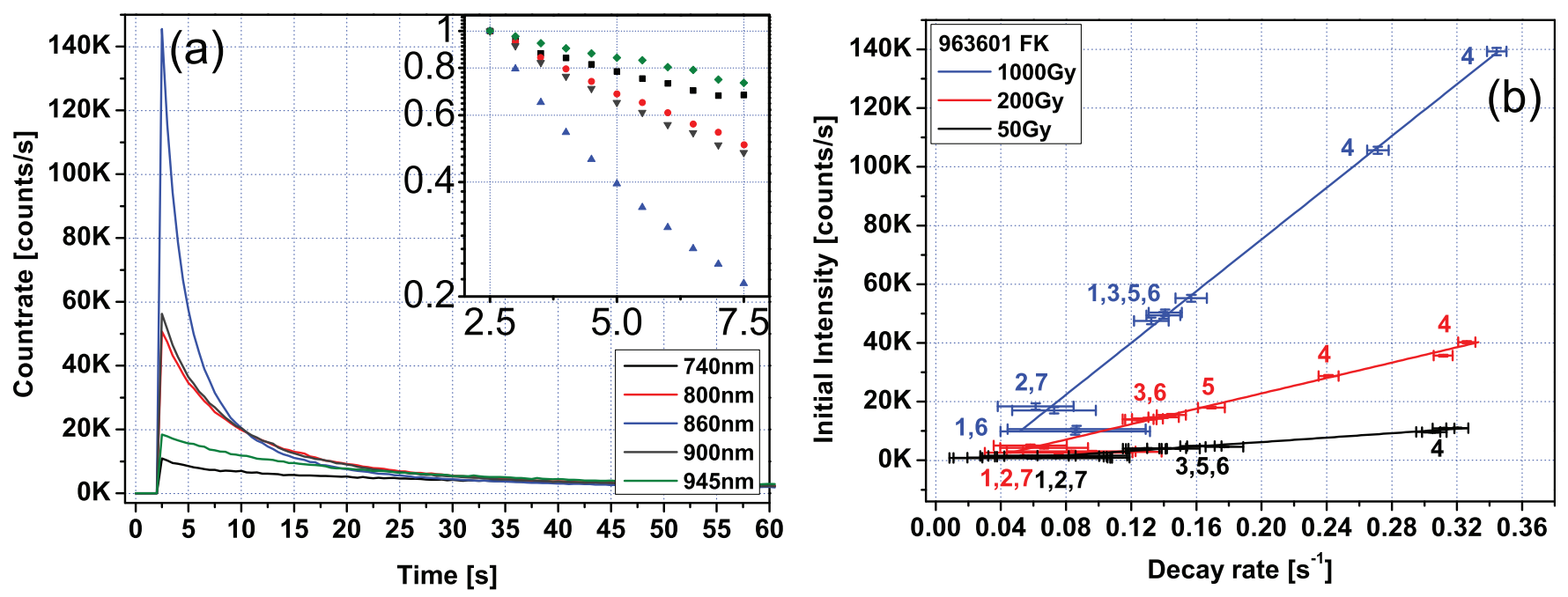

FIG. 8. (a) Representative OSL decay curves measured using different wavelengths from sample $963601 \mathrm{FK}$ after a given dose of $1000 \mathrm{~Gy}$. The inset shows normalized data in the first $5 \mathrm{~s}$ plotted on a log intensity scale; the color notation is the same as for the full OSL decay curves. (b) Initial intensity [ $\left.{ }^{-1}\right]$ plotted as a function exponential decay constant $\left[\mathrm{s}^{-1}\right]$ for one aliquot of $963601 \mathrm{FK}$ after a prior dose of 50,200 , or $1000 \mathrm{~Gy}$ and an optical stimulation at $30^{\circ} \mathrm{C}$. The offset from " 0 " on the $\mathrm{x}$-axis is for all curves are found to around $0.03 \mathrm{~s}^{-1}$. The number markers represent different stimulation wavelengths $(1=740 \mathrm{~nm}$, $2=760 \mathrm{~nm}, 3=800 \mathrm{~nm}, 4=860 \mathrm{~nm}, 5=900 \mathrm{~nm}, 6=910 \mathrm{~nm}, 7=945 \mathrm{~nm})$.

We conclude based on these data that the pIRIR and IRSL signal arise from the same trap.

\section{B. Dose response curves}

Each trap has a characteristic number density in the crystal and an electron capture cross-section. These characteristics are typically reflected as a single saturating exponential dose-response curve, where the exponential constant reflects the trapping cross-section. It is expected that different types of traps will have a different trapping cross-section because of the chemical nature of the defect or the surrounding charge environment of the trapping centre. Thus, same dose-response curves for different signals will most likely indicate that these signals arise from a single trap. On the other hand different dose response curves for different signals may not necessarily indicate that they arise from different traps, since there could be non-first order effects arising, for example, because of competition in the trapping or in the recombination process.

With this hypothesis we examine the dose response curves of the different signals using wavelength chosen to cover all of the ES peaks. The measurement method used was single aliquot regenerative (SAR) dose measurement ${ }^{26}$ and the protocol can be seen in Table II. It is expected that the SAR method corrects for any sensitivity changes prior to the measurement of the regeneration dose OSL. However this sensitivity correction method assumes that either there is no sensitivity change between the regeneration OSL and the test dose OSL or if there is a sensitivity change, then it is constant across the different SAR cycles. Fulfillment of these assumptions can be checked with a recycling ratio, which was found to be between $\sim 2$ and $5 \%$ for all our measurement. The dose response curves for range of different wavelengths at $30^{\circ} \mathrm{C}$ covering the ES peak, and the side bands over a $170 \mathrm{~nm}$ range for $963601 \mathrm{FK}$ and $240 \mathrm{~nm}$ for
R28 are shown in Fig. 6. The different dose response curves using the different wavelengths cannot be distinguished statistically for the samples 963601 FK (Fig. 6(a)) and R28 (Fig. 6(b)), suggesting that the same trap is sampled irrespective of the stimulation wavelength in our red-IR range.

We also applied the same analysis for comparison of the IR signals at different stimulation temperatures $(860 \mathrm{~nm}$; at $30^{\circ} \mathrm{C}, 230^{\circ} \mathrm{C}$, or $290^{\circ} \mathrm{C}$ ) and the pIRIR signals $(860 \mathrm{~nm}$, prior IR bleach at $30^{\circ} \mathrm{C}$ and pIRIR at $230^{\circ} \mathrm{C}$ or $290^{\circ} \mathrm{C}$ ) see Table III for the sequence protocol. The results are shown in Fig. 7.

The elevated measurements show that (a) all the high temperature curves whether IRSL and pIRIR (at 230 and $290^{\circ} \mathrm{C}$ ) are very similar and (b) the high temperature curves saturate at a lower dose compared to the IRSL measured at $30^{\circ} \mathrm{C}$.

Our DRC data convincingly show that the shape of the dose response curve is dependent on whether it is measured at low temperature and high temperature, and not so much on the wavelength or on whether there was a prior IR bleach or not.

The difference between the DRC's of the IR and the pIRIR signals cannot be explained by simply assuming that the high temperature signal is a mix between a less stable and a more stable trap ${ }^{14}$. In that case one would have expected the high temperature DRC to lie closer to the $50{ }^{\circ} \mathrm{C}$ IR curve than to the pIRIR curve, since the signal intensity of the pIRIR signal is less than $40 \%-80 \%$ of the corresponding high temperature IR signal; this is clearly not the case. The pIRIR DRC is either indistinguishable (e.g., at $290^{\circ} \mathrm{C}$ ) or very close (e.g., at $230^{\circ} \mathrm{C}$ ) to the corresponding high temperature DRC. Therefore, multiple trap hypotheses cannot explain these data.

One could also argue that the low temperature and high temperature IRSL signals arise from different traps, and the presence of prior IR is immaterial. Such a hypothesis can be 
ruled out since it is clear that the prior IR reduces the intensity of the pIRIR signal by $40 \%-80 \%$; therefore, IR bleaching has a significant impact on the system.

Equally, a simple single trap model cannot successfully explain the high temperature DRC data. On one hand it can be argued that the similarity of pIRIR and high temperature DRC's support the interpretation from the ES data that the same trap is involved in IRSL or pIRIR production irrespective of the stimulation temperature (Figs. 4 and 5). On the other hand, a single trap model cannot explain why there is a difference between the low temperature and high temperature DRC's. Our best explanation of these data is that the competition between retrapping and transport varies because of increased mobility at higher temperatures, and therefore the assumption of first order kinetics does not always apply. This interpretation suggests that the difference in the growth curve shape is not necessarily due to the presence of different traps but is rather a reflection of higher order processes. Nonetheless, one could reasonably assume that DRC's measured at same temperature can be compared across since the charge mobility is likely to be similar.

In summary the DRC data can be used to conclude that

(1) Growth curves at different wavelengths represent the same trap.

(2) An increase in stimulation temperature causes an earlier saturation in the growth curve. The difference between DRC's at room and higher temperatures (230 and $290^{\circ} \mathrm{C}$ ) does not imply different traps, but possibly a competing process.

(3) IRSL signals at elevated temperatures arise from the same trap as the corresponding pIRIR signals (also supported from the ES data in Fig. 2).

\section{Decay vs. initial intensity}

Although luminescence signal from feldspar arises from a complex recombination process, ${ }^{10,13,27}$ one can make a first-order approximation that during the infinitesimally small time at the beginning of light exposure, the decay rate is not limited by the hole density. Thus, the initial luminescence signal can be described as a change in the trapped electron population, i.e., $L_{0}=n_{0} b(\lambda)$, where $n_{0}$ is the initial trapped electron density (after irradiation) and $b$ is the detrapping probability, a product of stimulation light flux and the wavelength $(\lambda)$ dependent excitation cross section. For a single trap the term $n_{0}$ is constant since it is only a function of the prior dose. Thus, for a given dose the proportionality $L_{0} \propto b$ holds true irrespective of stimulation wavelength as long as a single trap is measured, the proportionality constant being $n_{0}$. Such a relationship can be used to test whether the initial luminescence signal measured using different wavelengths originates from a single trap (single proportionality) or multiple traps (multiple proportionalities). If different wavelengths are preferentially targeting different traps, then there should not exist a linear correlation between the different values of $L_{0}$ and $b$ obtained using the different wavelengths in the measurement range from 740 to $945 \mathrm{~nm}$. A similar approach has also been used earlier by Baril and Huntley ${ }^{22}$; however, they plot the $\sqrt{S_{0}}$ vs $L_{0}$, with $S_{0}$ being the initial rate of decrease in intensity.

In order to test the hypothesis we derived the decay constant $b$ and the initial intensity $L_{0}$ by using an exponential fit of the form $L=L_{0} e^{-b t}$ to the initial part (first $\sim 5 \mathrm{~s}$ ) of the OSL decay curves. For this analysis the OSL curves from the previous measurements for $963601 \mathrm{FK}$ sample are used and some of the decay curves are shown in Fig. 8(a). The results from fitted curves for all wavelengths are plotted in Fig. 8(b).

The data strongly suggests that the initial luminescence signal measured in the range from 740 to $945 \mathrm{~nm}$ is coming from the same trap regardless of stimulation wavelength, and as expected there is a increase in the slope with dose corresponding to an increase in the proportionality term $n_{0}$. Some of the curves were re-measured after a long time gap; these give different absolute values but the same ratio of the two parameters for a single wavelength. The small offset on the $\mathrm{x}-$ axis for all our data corresponds to a value of $\sim 0.03$. A similar observation has been made by Baril and Huntley. ${ }^{23}$ The reason for this offset is not clear and requires further investigations.

\section{CONCLUSIONS}

We conclude based on Red-IR excitation ( $700-1050 \mathrm{~nm})$ luminescence spectroscopy as a function of preheat temperature and stimulation temperature that the infra-red luminescence signal (IRSL) in K-feldspar arises from a single, deep dosimetric trap and not from multiple traps as proposed earlier. Similarly, the elevated temperature IRSL signal measured after a prior IR wash at room temperature (also called the post IR IRSL) is also concluded to arise from the same trap as the IRSL signal. The slight blue shift in the peak of the post IR excitation spectra of the order of $0-20 \mathrm{meV}$ reflects the thermal-diffusion energy barrier that needs to be overcome for the production the post IR IRSL; this barrier exists since all the nearby recombination sites are used up during the resonant IR bleach and, therefore, transport at higher energy band tail states is necessary for recombination.

The above conclusion is strongly supported by the data on (a) the dose response curves and (b) the shape of the OSL curve (initial intensity vs. the decay constant), measured over the entire spectral range; these data suggest that the initial signal is coming from the same trap irrespective of the stimulation wavelength. Based on these results we further conclude that the different peaks identified in the excitation spectra in previous work are different excited states of the dosimetric trap rather than different electron trapping states.

We also find a basic difference in the shape of the dose response curves for low and high temperature IRSL signals, irrespective of the prior IR bleach. This result probably reflects a competition in recombination/retrapping pathways because of increased electron mobility at higher temperatures; this effect cannot be attributed to any underlying trap structure.

\section{ACKNOWLEDGMENTS}

We would like to thank Dr. N.R.J. Poolton for kindly lending us the sample R28. 
${ }^{1}$ J.-P. Buylaert, M. Jain, A. S. Murray, K. J. Thomsen, C. Thiel, and R. Sohbati, "A robust feldspar luminescence dating method for middle and late pleistocene sediments," Boreas 41(3), 435-451 (2012).

${ }^{2}$ M. Jain, C. E. Andersen, L. Bøtter-Jensen, A. S. Murray, H. Haack, and J. C. Bridges, "Luminescence dating on Mars: OSL characteristics of Martian analogue materials and GCR dosimetry," Radiat. Meas. 41(7-8), 755-761 (2006).

${ }^{3}$ S. W. S. McKeever, D. Banerjee, M. Blair, S. M. Clifford, M. S. Clowdsley, S. S. Kim, M. Lamothe et al., "Concepts and approaches to in situ luminescence dating of martian sediments," Radiat. Meas. 37(4-5), 527-534 (2003).

${ }^{4}$ A. G. Wintle, "Anomalous fading of thermo-luminescence in mineral samples," Nature (London) 245(5421), 143-144 (1973).

${ }^{5} \mathrm{R}$. Visocekas, "Tunnelling radiative recombination in labradorite: Its association with anomalous fading of thermoluminescence," Nucl. Tracks Radiat. Meas. 10(4-6), 521-529 (1985).

${ }^{6} \mathrm{R}$. Visocekas, "Tunnelling in Afterglow: Its coexistence and interweaving with thermally stimulated luminescence," Radiat. Prot. Dosim. 100(1-4), 45-53 (2002).

${ }^{7}$ P. Morthekai, M. Jain, A. S. Murray, K. J. Thomsen, and L. Bøtter-Jensen, "Fading characteristics of martian analogue materials and the applicability of a correction procedure," Radiat. Meas. 43(2-6), 672-678 (2008).

${ }^{8}$ G. Hütt, I. Jaek, and J. Tchonka, "Optical dating: K-feldspars optical response stimulation spectra," Quaternary Sci. Rev. 7(3-4), 381-385 (1988).

${ }^{9}$ G. A. T. Duller, "Behavioural studies of stimulated luminescence from feldspars," Radiat. Meas. 27(5-6), 663-694 (1997).

${ }^{10} \mathrm{M}$. Jain and C. Ankjærgaard, "Towards a non-fading signal in feldspar: Insight into charge transport and tunnelling from time-resolved optically stimulated luminescence," Radiat. Meas. 46(3), 292-309 (2011).

${ }^{11}$ N. R. J. Poolton, R. H. Kars, J. Wallinga, and A. J. J. Bos, "Direct evidence for the participation of band-tails and excited-state tunnelling in the luminescence of irradiated feldspars," J. Phys.: Condens. Matter 21(48), 485505 (2009).

${ }^{12}$ M. Jain and A. Singhvi, "Limits to depletion of blue-green light stimulated luminescence in feldspars: Implications for quartz dating," Radiat. Meas. 33(6), 883-892 (2001).

${ }^{13}$ N. R. J. Poolton, K. B. Ozanyan, J. Wallinga, A. S. Murray, and L. BøtterJensen, "Electrons in feldspar II: A consideration of the influence of conduction band-tail states on luminescence processes," Phys. Chem. Miner. 29(3), 217-225 (2002).
${ }^{14} \mathrm{~B}$. Li and S.-H. Li, "Thermal stability of infrared stimulated luminescence of sedimentary K-feldspar,” Radiat. Meas. 46(1), 29-36 (2011a).

${ }^{15} \mathrm{~B}$. Li and $\mathrm{S} . \mathrm{H}$. Li, "Luminescence dating of K-feldspar from sediments: A protocol without anomalous fading correction," Quaternary Geochronol. 6(5), 468-479 (2011b).

${ }^{16}$ K. J. Thomsen, A. S. Murray, M. Jain, and L. Bøtter-Jensen, "Laboratory fading rates of various luminescence signals from feldspar-rich sediment extracts," Radiat. Meas. 43(9-10), 1474-1486 (2008).

${ }^{17}$ J.-P. Buylaert, A. S. Murray, K. J. Thomsen, and M. Jain, "Testing the potential of an elevated temperature IRSL signal from K-feldspar," Radiat. Meas. 44(5-6), 560-565 (2009).

${ }^{18}$ K. J. Thomsen, A. S. Murray, and M. Jain, "Stability of IRSL signals from sedimentary K-feldspar samples," Geochronometria 38(1), 1-13 (2011).

${ }^{19}$ A. S. Murray, J.-P. Buylaert, K. J. Thomsen, and M. Jain, "The effect of preheating on the IRSL signal from feldspar," Radiat. Meas. 44(5-6), 554-559 (2009).

${ }^{20}$ C. Ankjærgaard, M. Jain, R. Kalchgruber, T. Lapp, D. Klein, S. W. S. McKeever, and A. S. Murray, "Further investigations into pulsed optically stimulated luminescence from feldspars using blue and green light," Radiat. Meas. 44(5-6), 576-581 (2009).

${ }^{21} \mathrm{~L}$. Bøtter-Jensen, K. J. Thomsen, and M. Jain, "Review of optically stimulated luminescence (OSL) instrumental developments for retrospective dosimetry," Radiat. Meas. 45 253-257 (2010).

${ }^{22}$ C. Thiel, J.-P. Buylaert, A. Murray, B. Terhorst, I. Hofer, S. Tsukamoto, and M. Frechen, "Luminescence dating of the Stratzing loess profile (Austria) - Testing the potential of an elevated temperature post-IR IRSL protocol," Quaternary Int. 234(1-2), 23-31 (2011).

${ }^{23}$ M. R. Baril and D. J. Huntley, "Optical excitation spectra of trapped electrons in irradiated feldspars," J. Phys.: Condens. Matter 15(46), 8029-8048 (2003).

${ }^{24}$ D. C. W. Sanderson, "Fading of thermoluminescence in feldspars: Characteristics and corrections," Int. J. Rad. Appl. Instrum. D 14(1-2), 155-161 (1998).

${ }^{25}$ G. A. T. Duller and L. Bøtter-Jensen, "Luminescence from potassium feldspars stimulated by infrared and green light," Radiat. Prot. Dosim. 47(1-4), 683-688 (1993).

${ }^{26}$ A. S. Murray and A. G. Wintle, "Luminescence dating of quartz using an improved single-aliquot regenerative-dose protocol," Radiat. Meas. 32(1), 57-73 (2000).

${ }^{27} \mathrm{C}$. Ankjærgaard and M. Jain, "Optically stimulated phosphorescence in orthoclase feldspar over the millisecond to second time scale," J. Lumin. 130(12), 2346-2355 (2010). 